\title{
Recursos en Internet sobre el libro antiguo en Europa
}

\author{
Jesús de Diego Erles \\ Esperanza Velasco de la Peña \\ Universidad de Zaragoza
}

\subsection{Resumen}

Se realiza un directorio de los recursos que existen en la red sobre libro antiguo en Europa, incluyendo catálogos de bibliotecas, asociaciones profesionales y de libreros, páginas de museos y librerías o las listas de distribución y de noticias.

Palabras clave: Historia del libro. Libro antiguo. Internet. World Wide Web.

\subsection{Abstract}

A directory of Internet information sources on old books in Europe is offered. It includes databases and catalogues, distribution lists, news and web pages related to libraries, professional associations of librarians and booksellers, and museus.

Keywords: History of book. Old books. Internet. World Wide Web.

\section{Introducción}

Es un tópico habitual insistir una vez más en la transcendencia social del fenómeno Internet. El ámbito de la cultura, y en concreto, el referido al patrimonio bibliográfico no podía estar ajeno a esta revolución tecnológica. Los centros bibliotecarios han asumido la integración de la Red en su trabajo, descubriendo una serie de aplicaciones que permiten ofrecer servicios novedosos a sus usuarios. La web es hoy en día el sistema más aceptado para organizar y servir la información. El libro antiguo ha sido uno de los más favorecidos en este aspecto, ya que las tecnologías de Internet favorecen su difusión, tanto a tráves de catálogos como por textos integra o parcialmente digitalizados. Incluso en el caso de la digitalización no sólo se esta facilitando un acceso directo al texto, sino que se esta contribuyendo a su preservación y conservación al evitar la manipulación del original. 
Existen en la web, además de catálogos de colecciones bibliotecarias, otras sedes dedicadas al libro antiguo, desde asociaciones profesionales hasta museos, pasando por librerias especializadas. Todas ellas nos remiten a enlaces de interés para el tema que nos ocupa.

Igualmente la Red nos permite el uso de herramientas, como las listas de distribución o las noticias, que nos posibilitan estar en contacto con el libro antiguo.

Sin querer ser exhaustivos y teniendo en cuenta la situación cambiante de Internet, nuestro trabajo quiere poner en evidencia aquellas URL,s que consideramos de mayor relevancia en el universo del libro antiguo. Somos conscientes de que en el momento de poner punto final a nuestra ponencia estarán surgiendo nuevas sedes, algunas habrán cambiado su URL e incluso otras habrán desaparecido.

\section{Catálogos de bibliotecas}

Cada vez son más las bibliotecas con fondo antiguo que vuelcan su contenido a Internet, facilitándonos de este modo el acceso al patrimonio bibliográfico existente. Los Catálogos Colectivos son la herramienta más útil para ello, ya que permiten la búsqueda varias bibliotecas a la vez. En este apartado, además de dichos catálogos, recogemos otras bibliotecas universitarias y municipales que cuentan con fondo antiguo impreso.

\subsection{The Hand Printed Book (HPB)}

HPB es la base de datos creada por iniciativa del CERL (Consortium of European Research Libraries) <http://www.cerl.org/>, que contiene libros impresos en Europa anteriores a 1830. El Consorcio de Bibliotecas de Investigación Europeas fue fundado en 1992. En la actualidad hay 50 instituciones de 25 países asociadas y cuenta con los ficheros de 10 bibliotecas: Kungliga Biblioteket de Estocolmo, Biblioteca Nacional y Universitaria de Zagreb, ICCU de Roma, Biblioteca Nacional de Escocia, Biblioteca Nacional de Francia, Biblioteca del Estado de Baviera de Munich, British Library de Londres (K17 y ISTC), Biblioteca Nacional y Universitaria de Ljubljana, en Eslovenia, y Koninklijke Bibliothek de la Haya. La próxima biblioteca es la Nacional de Madrid. El acceso a la base de datos por ahora está limitado a los miembros del Consorcio <http://eureka.thames.rlg.org>.

\subsection{Alemania}

Existen en Alemania importantes catálogos colectivos con acceso a fondos correspondientes al libro antiguo, cuya información esta, generalmente en alemán e inglés: 
La DBI-LINK, cuya sede esta en la URL <http: www. dbilink.de>, es una gran base de datos que da acceso al Catálogo Colectivo conocido como VK97 en el que participan alrededor de 3.000 bibliotecas. Desde aquí podemos entrar también en el Berlin OPAC, catálogo de las bibliotecas de la ciudad de Berlín. Ambos cuentan con importantes fondos de libro antiguo.

Hay que señalar que se puede acceder a la consulta de forma gratuita, como invitado, o bien, mediante pago, para beneficiarse de otros servicios bibliotecarios. La URL es: 〈http://dbix01.dbi-berlin.de:6100/DBI/login.html>.

La Universidad de Karlsruhe <http://www.ubka.uni.karlsruhe.de> ofrece el Catálogo virtual, Karlsruher Virtueller Katalog, desde el que podemos hacer una búsqueda múltiple en varios catálogos de países germano parlantes, Reino Unido y Estados Unidos, así como en las Bibliotecas Nacionales de Francia y España. Su URL es: 〈http://www.ubka.uni-karlsruhe.de/hylib/en/kvk.html>.

Tampoco hay que olvidar la Gateway Z3950 de las bibliotecas alemanas Deutsche Bibliothek <http://z3950gw.dbf.ddb.de/> que también reagrupa varios catálogos, alguno de ellos con fondo antiguo.

IPAC (Image Public Access Catalogue) es la base de datos que contiene las fichas catalográficas de la Berlin, Zentral-und Landesbibliothek. $<$ http://ipac.zlb.de/>

\subsection{Austria}

El Catálogo Colectivo de las Bibliotecas de Austria Online-Katalogen des Österreichischen Bibliothekenverbundes, comprende la Österreichische Nationalbibliotek, de Viena, y otras bibliotecas universitarias, tanto de Viena como de Graz, Innsbruck, Klagenfurt, Leoben, Linz, Mödlinga y Salzburg, cuya dirección es <http://www.bibvb.ac.at/verbund-opac.htm>.

Otra dirección interesante es la que corresponde a la reproducción de las fichas catalográficas mecanografiadas de la Österreichische Nationalbibliotek, de Viena, con las obras de los años 1501 a 1929. Su URL es <http://www.onb.ac.at/index.htm>. Desde ella podemos aceptar una de estas tres opciones: la consulta del catálogo a partir del índice de autoridades (<http://www.onb.ac.at/online_s/katzoom.htm>); por materias (<http://euler.onb.ac.at/cgi-katzoom/katzoom.pl?katalog=4〉); o bien ir al catálogo en línea.

\subsection{Bélgica}

El catálogo colectivo de Bélgica (CCB) es accesible, por el momento en CDROM, aunque próximamente esta previsto el volcado en DVD. Toda la información sobre el catálogo colectivo belga y las bibliotecas que lo ponen podemos hallarla en la URL: 〈http://www.libis.kuleuven.ac.be/libis/ccb/> 
La red LIBIS-NET, agrupa 16 bibliotecas de las universidades de Bruxelas, Namur y la católica de Lovaina. Comprende un buen número de libros antiguos, la mayoría procededente de Lovaina. Su URL es <http://www.libis.kuleuven.ac.be/libis/index.html>

La sede de la Universidad de Amberes <http://lib.ua.ac.be/> da acceso a través de la red ANET a una red de bibliotecas universitarias y especializadas, sitas en la región de Amberes. La búsqueda se hace por medio de la Unión de Catálogos Zebra.

Otra biblioteca a destacar con fondos antiguos es la Biblioteca Real $<$ http://www.kbr.be/>, cuyo catálogo no esta en este momento disponible en la red.

\subsection{Dinamarca}

La Biblioteca Real de Dinamarca <http://www.kb.dk/> cuenta con un importante fondo antiguo al que accedemos por el catalogo REX.

\subsection{España}

El Catálogo Colectivo del Patrimonio Bibliográfico Español (PABI) ha sido elaborado por el Ministerio de Educación y Cultura y por las Comunidades Autónomas, y tiene como objeto la descripción de las bibliotecas públicas y privadas del Patrimonio Bibliográfico. Su URL es $<$ http://www.mcu.es/ccpb/index.html>.

Otros catálogos españoles importantes son:

- BU(Catalogo Colectivo de las Universidades de Cataluña) <http:// www.cbuc.es/ccuc/>

- RUECA (Catálogo de bibliotecas universitarias que utilizan ABSYS). <http:// www.baratz.es/RUECA/>

- RUEDO (Catálogo de bibliotecas universitarias que utilizan Dobis/Libis) <http://info.uned.es/biblioteca/ruefdo.htm>

- Biblioteca Nacional de Madrid: 〈http://www.bne.es>

- Biblioteca de Cataluña de Barcelona: <http.// www.gencat.es/bc/>

Además, en la sede de la Diputación Provincial de Zaragoza encontramos BAMBA (Bibliografía Aragonesa Mecanizada de las Bibliotecas de Aragón), es un catálogo informatizado de las publicaciones aragonesas o de tema aragonés que existían en algunas Bibliotecas de Aragón en 1986. Su URL es <http://ariel.unizar.es/dpz/bamba/default.html> 


\subsection{Finlandia}

Destaca HELKA, catalogo de las bibliotecas universitarias de Helsinki $<$ http://orm2.lib.helsinki.fi/en/>.

\subsection{Francia}

La sede de la Biblioteca Nacional de Francia no da todavía acceso al acervo bibliográfico que se encuentra depositado en departamentos especializados como las bibliotecas Richelieu, Opera y Arsenal. Su URL es <http://www.bnf.fr>,

Otro proyecto de gran envergadura es el Catálogo Colectivo de Francia <http://www.ccfr.bnf.fr/>, todavía en proceso de realización.

La Bibliothèque Universitaire de Medicine (BIUM) ha digitalizado el catalogo antiguo que contiene unas 337.000 fichas, manuscritas, de obras impresas desde 1478 hasta 1952. Su URL es <http://www.bium.univparis5.fr/acc_gen.htm>

Entre las bibliotecas municipales con importante fondo antiguo hay que destacar las de:

- Lyon: <http://www.bm-lyon.fr>

- Besançon: <http://www.besancon.com/ABSYS/>

- Clermont-Ferrand: 〈http://bmiu.univ.bpclermont.fr>

- Blois: <htttp://ville-blois.fr/bibliotheques-blois/default-internet.asp

\subsection{Gran Bretaña}

Gran Bretaña aporta varias direcciones de interés para nosotros, siendo las más relevantes de todas :

- British Library de Londres <http://portico.bl.uk>. Contiene el OPAC 97, catálogo en línea de la British Library, cuya URL es 〈http://opac97.bl.uk>. Entre las diferentes opciones que ofrece el formulario de búsqueda elegimos Older reference material (to 1975 only), por ser la que se adecúa a nuestras necesidades. Además la British ofrece otros catálogos para el libro antiguo consultables previo pago como son: Incunable Short Title Catalogue (ISTC) y el Eighteenth Century Short Title Catalogue (ESTC).

- CURL (Consortium of University Research Libraries) <http://curl.ac.uk> es la agrupación de bibliotecas británicas que soportan el protocolo Z39.50, del que nace COPAC, catálogo colectivo de las siguientes bibliotecas universitarias: Cambridge University, Edinburg University, Glasgow University, Imperial College, LeedsUniversity, Manchester University, Nottingham University, Trinity College Dublin, University College 
London y University of London Library. Está previsto que pronto se unan otros doce centros más. Su URL es <http://copac.ac.uk/copac〉.

Tenemos además los fondos de las bibliotecas escocesas como:

- La National Library of Scotland's, cuya URL es: 〈http://www.nls.uk/ >.

- La Universidad de Napier tiene consultables, via Web o Telnet:, las bibliotecas de su Campus. Como página web accedemos desde la URL <http://www.napier.ac.uk/depts/library/homepage.htm>, y luego pulsaremos Resources y luego la opción Catalogues.

- La biblioteca de la Universidad de St. Andrews con su catálogo SAULCAT: <http://www-library.st-and.ac.uk/>. Una vez en el pulsaremos Special Collections.

Otras bibliotecas con notable fondo antiguo son:

- La National Library of Wales: 〈hhttp://www.llgc.org.uk/>

- La University Library de Cardiff : <http://www.cf.ac.uk/uwcc/infos/information//index.html>

- La National Library of Art del Victoria and Albert Museum de Londres: $<$ http://www.nal.vam.ac.uk/>

- Todas la relación de bibliotecas del Campus de la Universidad de Oxford son visualizables desde <http://www.lib.ox.ac.uk/libraries/> . Por medio de esta sede accedemos a OLIS, Catálogo Colectivo de estas bibliotecas universitarias, cuya URL es <http://saturn.olis.ox.ac.uk:8000/>.

- También hay que tener en cuenta la Reading University Library, cuya URL es: <http://www.rdg.ac.uk/SerDepts/vl/home.html>, y que ofrece el catalogo Unicorn al que entramos como invitados, con la opción Guest.

\subsection{Holanda}

- PiCarta es una gran base de datos que integra al Catálogo Colectivo Holandés (Nederlandse Centrale Catalogus, NCC). Se trata de un servicio restringido para todas las bibliotecasque forman parte de este proyecto, sin embargo existe en la misma sede una demo de libre acceso con unas 40.000 referencias. <http://www.pica.nl/en/>

- La Koninklijke Bibliotheek de la Haya <http://www.konbib.nl/>

- La Biblioteca de la Universidad de Nijmegen : <http://opc.ubn.kun.nl/> o $<$ http://www.kun.nl/ubn/>

- La Biblioteca de la Universidad de Utrecht: <http://www.ubu.ruu.nl/uulhome> 


\subsection{Italia}

Nos encontramos también con los siguientes recursos:

- Catalogo Colectivo. El Istituto Centrale per il Catalogo Unico delle Biblioteche Italiane e per le Informazioni Bibliografiche (ICCU), dependiente del Ministero per i Bene Culturale y Ambientale, es el encargado de impulsar el catálogo colectivo del libro italiano existente en bibliotecas públicas y privadas. Toda la información de este Catálogo y de las bibliotecas participantes se encuentra en 〈http://www.sbn.it>. Para acceder a la base de datos el usuario pulsará la opción OPAC dell'Indice SBN. Dentro ya del OPAC se deberá activar la correspondiente a los libros impresos antiguos, es decir, anteriores a 1830.

- EDIT16 es también un proyecto promovido por el Istituto Centrale per il Catalogo Unico en el que participan un total de 1.200 bibliotecas, entre las que se incluyen las de la República de San Marino y El Vaticano, además de las italianas. EDIT 16 consiste en la realización de un censo de las ediciones del siglo XVI impresas en Italia o fuera de ella cuya lengua sea el italiano. Por el momento hay 50.000 noticias bibliográficas volcadas en una base de datos que recogen la identificación de la edición (autor, titulo, impresor, editor, marca tipográfica digitalizada, etc.). La información relativa al proyecto EDIT 16 la encontramos en: $<$ http://edit16.iccu.sbn.it/iccu.htm>, pero para acceder a la base de datos la URL es <http://edit16.iccu.sbn.it/imain.htm>

- IRIS (Associazione di Biblioteche Storico Artistiche e Umanistiche a Firenze), es una asociación de bibliotecas florentinas, incardinadas en torno al Renacimiento en todos sus aspectos, incidiendo sobre todo en el Renacimiento italiano. Los miembros componentes son: Biblioteca Berenson - Villa I Tatti, Biblioteca della Soprintendenza ai Beni Artistici e Storici - Firenze, Biblioteca della Fondazione di Studi di Storia dell'Arte "Roberto Longhi", Biblioteca dell'Istituto Nazionale di Studi sul Rinascimento, Biblioteca dell'Istituto Universitario Olandese di Storia dell'Arte, y la Biblioteca dell'Opificio delle Pietre Dure. La sede da información de cada una de las bibliotecas citadas, con su historia, su colección, horario, servicios que ofrece, así como el enlace con la URL correspondiente a cada institución de la que dependen. A su vez la sede indica otros enlaces de interés para el mundo bibliotecario. El catálogo colectivo de todas ellas, con cerca de 130.000 títulos, entre los que encontramos un buen número de libros antiguos, tiene acceso a Internet mediante plataforma Aleph 500. La URL es <http://www.iris.firenze.it/> 
- URBS (Unione Romane delle Biblioteche Scientifiche) es una red que contiene 14 bibliotecas con fondos antiguos, entre ellas, parte de la Biblioteca Vaticana. 〈http://www-urbs.vatlib.it>

- Desde la Biblioteca Nazionale Centrale di Firenze llegamos al Catálogo Palatino que consiste en el proyecto de digitalización de 225.000 fichas mecanografiadas, correspondientes a las obras de los siglos XVI y XIX de la Biblioteca Palatina de Lorena y de otros fondos que ingresaron en ella (Nencini, Passerini, Targioni Tozzetti, Filippini e Miscellanea Capretta). Hoy en día constituyen, junto al fondo Magliabechiano, el núcleo del Fondo Histórico de la Biblioteca Nacional de Florencia. Por el momento únicamente están escaneadas y puestas en la Red las fichas correspondientes a la A.

- Otro proyecto en curso promovido por la Biblioteca Nacional de Florencia es el Catálogo Sacconi. Se trata de la reconversión electrónica de este catálogo del siglo XIX, mediante su digitalización. La URL para entrar en este $\begin{array}{llllll}\text { ptoyecto } & y & \text { en } & \text { el anterior }\end{array}$ <http://www.bncf.firenze.sbn.it/progetti/index.html>

- Il Seicento, catálogo del Istituto Veneto di Scienze, Lettere ed Arti (Venecia), presenta cerca de 800 obras del siglo XVII con una descripción muy detallada. <http://www.ivsla.unive.it/seicento/seicento.htm>

- La Biblioteca del Capitolo Metropolitano di Milano que posee un rico patrimonio bibliográfico tiene como proyecto volcar en Internet el catálogo de sus 76 incunables. Hasta este momento son 10 los reflejados en el Catalogo Incunaboli. Esta biblioteca milanesa tiene además en su haber 1000 obras correspondientes al siglo XVI. Su URL: <http://www.promo.it/novantiqua/milano/catalogo.htm>

- La Biblioteca Biblioteca Municipal Renato Fucini, de Empoli, cuenta con 30.000 ediciones de fondo antiguo, entre las que encontramos 5 incunables y 720 ejemplares del siglo XVI. En ella están depositados, entre otros, lo fondos Giovanni Marchetti, Antonio Salvagnoli Marchetti, Giuseppe Tassinari, Convento dei Cappuccini y Convento di Santa Maria a Ripa. Esta biblioteca con la colaboración del Ministero dell'Ufficio Biblioteche, Beni Librari e Attività Culturali della Regione Toscana esta llevando a cabo distintos proyectos referentes al libro antiguo. Para más información puede consultarse en <http://www.comune.empoli.fi.it/biblioteca/antiche.htm>

- Es ya una realidad el Catalogo delle edizioni del Cinquecento, en CDROM, consultable en línea, con las ediciones del siglo XVI pertenecientes a esta biblioteca. Las noticias bibliográficas están asociadas a imágenes de 
frontispicios, marcas tipográficas, ex libris y notas manuscritas de los respectivos

ejemplares.

<htp://www.comune.empoli.fi.it/biblioteca/cdrom.htm>

\subsection{Portugal}

El catálogo colectivo portugués accesible mediante Internet se llama PORBASE, y recoge los catálogos de la Biblioteca Nacional, que actúa también como coordinadora, y de otras 143 bibliotecas más, tanto públicas como privadas, de las que la mayoría son universitarias. Por lo que respecta al libro antiguo, destacan los conservados por la Biblioteca Nacional, con 1.500 incunables, y un importante número de ejemplares de los siglos XVI y siguientes. A través de la URL < http://www.ibl.pt> tenemos acceso a la sede de la Biblioteca Nacional de Portugal, y desde aquí podemos acceder a PORBASE por la interfaz WWW <http://sirius.bn.pt/ >.

\subsection{República Checa}

Destacamos la Biblioteca Nacional de Praga, con una URL:<http://www.nkp.cz/>. Los catálogos antiguos están mecanografiados y sus fichas catalográficas son accesibles por la RED mediante la URL: $<$ http://katif.nkp.cz/d >. Para visualizarlos se requiere un conector TIFF.

\subsection{Suecia}

Existe el catálogo Colectivo LIBRIS, que incluye la Biblioteca Real de Estocolmo. URL: <http://www.libris.kb.se/.>

\subsection{Suiza}

Podemos señalar las siguientes aportaciones:

- RERO es la red formada por varias bibliotecas de áreas francófonas e italo parlantes, sitas en la Suiza Occidental, cuya URL es <http://www.rero.ch>.

- REBUS es la red de bibliotecas de la Suiza de habla alemana que utilizan la interfaz WWW SIBIL. Su URL es <http://www.rebus.unibe.ch>.

- Biblioteca Nacional Suiza, en Berna <http: www.snl.ch/>.

\subsection{El Vaticano}

En la Biblioteca Apostólica Vaticana nos encontramos con un millón y medio de libros, de los cuales 8.000 son incunables. Su acceso es vía Telnet. Su dirección es <telnet://libr6k.vatlib.it>.

\section{Bibliografias}

Las Bibliotecas antigua y nueva de escritores aragoneses de Latassa aumentadas y refundidas en forma de diccionario bibliográfico-biográfico por don

Scire. $5: 1$ (en.-jun. 1999) 91-103. 
Miguel Gómez Uriel (Zaragoza, mprenta de Calisto Ariño, 1884-1886, 3 vols.) son accesibles por Internet desde marzo de 1999. Esta edición electrónica ha sido realizada por Manuel José Pedraza Gracia, José Angel Sánchez Ibáñez y Luis Julve Larraz y es accesible mediante la sede de la Facultad de Filosofía y Letras de Zaragoza su URL es: 〈http://fyl.unizar.es/Latassa/Latassa.html>

\section{Asociaciones profesionales}

En todas estas sedes se encuentra noticias de la asociación y sus fines, así como de su actividad, ofreciendo la mayoría enlaces de interés para el libro antiguo. Cabe destacar entre ellas:

- ABEI (Ass. Bibliotecari Ecclesiastici Italiani), Asociación de bibliotecarios eclesiáticos italianos <http://utenti.tripod.it/novantiqua2/abei/index.htm>

- Sección de Rare Books and Manuscripts de la IFLA, en la que se incluye planes y conferencias. 〈http://www.ifla.org/VII/s18/srbm.htm>

- The Library Association: Rare Books Group (Gran Bretaña), asociación de bibliotecarios y otros profesionales responsables de colecciones de libros antiguos. Es una de las sedes más completas e interesantes. <http://venus.las.ox.ac.uk/rbg/Rare_books.htm>

- The Bibliographical Society de Gran Bretaña, fundada en 1892, cuya URL es: 〈http://bibsoc.org.uk/>

\section{Asociaciones de Libreros}

- Ligue Internationale de la Librairie Ancienne. Se trata, como su nombre indica, de la unión internacional de asociaciones de libreros. URL=<http://ilab.org/ilab/french.html >

- Bibliorare. Association pour la recherche du Livre et document rares et precieux. Se trata de una asociación francesa. Puesta en la red el 29 de marzo de 2000. URL=<http://perso.wanadoo.fr/bibliorare/>

\section{Museos sobre la imprenta}

Bajo este epígrafe reunimos las sedes de los museos dedicados a la imprenta. En líneas generales podemos decir que la información que se encuentra en ellas se refiere a los horarios de apertura, dando una reseña más o menos breve del contenido que albergan, ilustrado con fotografías. Ello se presenta en la lengua del país de origen y en inglés, excepto en el caso del Museo de Guttenberg. La mayoría de las sedes incluye además, como valor añadido, enlaces de interés al tema que nos ocupa. 
- Gutenberg, en Mainz (Alemania). Dedicado al padre de la imprenta. <http://www.uni-mainz.de/UniInfo/Stadt/Museen/gutenberg.html>

- Plantin-Moretus, en Amberes (Bélgica). Hay que destacar la importante colección libraria que alberga, así como las antiguas prensas conservadas. 〈http://www.dma.be/cultuur/museum_plantinmoretus/index_eng.html >

- Bodoni, en Parma (Italia). Dedicado a Giambattista Bodoni, tipógrafo italiano del Setecientos. 〈http://www.comune.parma.it/turismo/mu-bo.htm>

- Musée de l'imprimerie de Lyon, en Francia. No podía faltar un museo de la imprenta en una ciudad que tanta importancia tuvo para el esplendor de la imprenta en la Edad Moderna. Tiene una pega, cuesta mucho de cargar. <http://www.bm-lyon.fr/musee/imprimerie.htm>

- The Virtual Museum of Printing Press, en Oporto (Portugal). Se trata de un proyecto virtual sobre la imprenta iniciado por AMI (Asociación del Museo de la Imprenta), cuyo fin es promocionar la historia de la imprenta y su patrimonio en todo el mundo. <http://www.imultimedia.pt/museuvirtpress/index_i.html>

- Melbourne Museum of Printing, Australia. Museo dedicado a la imprenta mecánica. Una de las mejores sedes, pese a que no esta terminada, con una completísima información sobre las piezas que componen este museo. Recogemos aquí su URL porque completaría la historia de la imprenta hasta nuestros días, con todas sus técnicas. <http://home.vicnet.net.au/ typo/outside.htm>

- Robert C. Williams American Museum of Papermaking, EEUU, sito en el

- Institute of Paper Science and Techbology de Atlanta (Georgia). Muy importante para la historia del papel. URL $=\langle$ http://www.ipst.edu/amp/>

- The National Print Museum of Ireland, en Dublin. Aunque esta dedicado a la imprenta mecánica, desde sus inicios, hemos querido reseñar este museo por la importancia que tiene para la historia de la imprenta irlandesa, de la que recoge datos desde el periodo incunable. <http://ireland.iol.ie/ npmuseum/>

\section{Listas de distribución y de noticias}

Otros de los recursos de información sobre el libro antiguo son las listas de distribución -vía correo electrónico- y los grupos de noticias, foros constituidos generalmente sobre un único tema. Entre las más importantes cabe citar: 


\subsection{Francia}

- BIBLIOPHILE, fr.rec.bibliophile. Grupo de discusión sobre el libro antiguo creado por Bertrand Hugonnard-Roche.

- Ex-Libris, Laval - Lista de distribución dedicada principalmente a la venta de libros antiguos. <http://www.lexlibris.com>

- LIVANTIC. Lista de distribución consagrada a la compraventa y cambio de libros antiguos. 〈http://www.livantic.com >

- Syndicat nationale de la librarie antigue et moderne. Lista de distribución que al igual a que a las anteriores se dedica fundamentalmente a la compraventa de libro. <http://www.Slam-Livre.fr/>

\subsection{Inglaterra}

- LIS- LIBHIST, lista de distribución sobre la historia del libro y de las bibliotecas. <http: /www.mailbase. ac.uk/ lists/ lis.libhist />

- LIS-RAREBOOKS, lista de distribución relativa al libro antiguo en general. <http: /www.mailbase. ac.uk/ list/ lis-rarebools/>

\section{Ornamentos tipográficos}

En Francia, Passe-Partout es un banco de datos internacional con ornamentos tipográficos, localizado en el Centro Informático de la Universidad de Laussanne, en Suiza.

En este momento participa la biblioteca cantonal y universitaria de Laussane con dos bases de datos de adornos tipográficos del Siglo de las Luces: Vignette que contiene los de los impresores que ejercían su actividad en esta ciudad y Fleuron que corresponde a los ornamentos de los tipógrafos de la Suiza francesa.

Sus respectivas URLs son:

- 〈http://dbserv-bcu.unil.ch/tango/todai/vignette.qry>

- 〈http://dbserv-bcu.unil.ch/tango/todai/fleuron.qry>

En la Facultad de Filosofía de la Universidad de Lieja, en Bélgica, existe otra base de datos Moriane también con ornamentos del Setecientos, cuya URL es: <http://www.ulg.ac.be/moriane/>

En Italia, el Archivio Umanistico Rinascimentale Bolognese (ARUB), dependiente de la Universidad de Bolonia, está llevando a cabo un proyecto por medio del laboratorio bibliológico que consiste en la elaboración de una base de datos de caracteres tipográficos de incunables boloñeses. Puede consultarse en la siguiente URL: 〈http://arub.unibo.it/lb/typebase.html> >. 
También en la Universidad inglesa de Bristol, de manera experimental, Earle Randall está a cargo de una base de datos que contiene las marcas tipográficas, portadas, etc. de algunos libros provenientes de la biblioteca de esta Universidad, con URL, http://www.bris.ac.uk/Depts/ContEd/E.Randall/index.html

\section{Librerías}

Cada vez son más librerías dedicadas a la venta del libro antiguo presentes en la Red. Todas ellas ofrecen sus catálogos en línea además de otros enlaces de interés. En Europa, principalmente en Francia y Gran Bretaña, cabe destacarlas siguientes URL,s:

- http://www.bouquins.net/

- http://www.abebooks.com/home/PHILHILLBKS/

- http://www.antiqbook.com/delezenaar/

- http://www.galaxidion.com/godon/

- http://www.rarebooks.ch/

- http://www.slam-livre.fr/latude/ 